Pacific Journal of Mathematics

ON THE INTERSECTION OF REGRESSIVE SETS

eph Barack and thomas Graham McLaughlin 


\title{
ON THE INTERSECTION OF REGRESSIVE SETS
}

\author{
J. BARBaCK AND T. G. McLaughlin
}

Let $A$ and $B$ be regressive isols and let $\alpha \in A$ and $\beta \in B$. Let $Y$ be the isol containing the set $\alpha \cap \beta$. We study some basic features of the isol $Y$, and features of $Y$ in the special case the sum $A+B$ is regressive. We also show that there is a large variety of regressive isols $A$ and $B$ for which the values of $Y$ that are associated, in the above way, are all finite.

1. Introduction. The results presented in this paper developed from an interest in sets that are the intersection of two regressive sets, and in the isols that contain such sets. (We shall agree that finite sets are both regressive and retraceable.) The following results are known: the intersection of two retraceable sets is retraceable (this is implicit in [7]), but the intersection of a cosimple retraceable set and a cosimple regressive set need not be regressive [1]. On the other hand ([2]) the intersection of any two regressive sets must, if infinite, have an infinite regressive subset. Several of the facts that are developed here were motivated by the following result from [5], which we state in terms of isols:

Theorem (Dekker). Let $A$ be a regressive isol and let $\alpha, \beta \in A$. Then $\alpha \cap \beta$ is a regressive set.

We shall prove one generalization of the above theorem. A part of our result gives the following: if $\alpha$ and $\beta$ are regressive sets that belong to isols whose sum is regressive, then $\alpha \cap \beta$ is also a regressive set.

For regressive isols $A$ and $B$, let $A \cap B$ denote the collection of all isols that contain a set which is the intersection of a set in $A$ with a set in $B$. Various properties of the collection $A \cap B$ are derived in the paper. One, in particular, is the following: given any cosimple regressive isol $A$, there exists an infinite regressive isol $B$ such that all isols in $A \cap B$ are finite.

We will assume that the reader is familiar with the basic notions in the theory of isols and in the theory of regressive isols. Certain special notions, like T-retraceability, are important in the paper, and these will be defined in the sequel. If $\alpha$ is an isolated set then $[\alpha]$ will denote the isol to which $\alpha$ belongs. We recall that sets $\alpha$ and $\beta$ are recursively equivalent if there exists a oneto-one partial recursive function $f$ defined on $\alpha$ and with $f(\alpha)=\beta$. 
We write $\omega$ for the set $(0,1,2, \cdots)$. If $f$ is a partial function from a subset of $\omega$ (or a subset of $\omega^{2}$ ) into $\omega$, then $\delta f$ will denote the domain of $f$.

We let $j(x, y)$ denote the familiar recursive pairing function $x+1 / 2(x+y)(x+y+1)$ that maps $\omega^{2}$ one-to-one and onto $\omega$. The degree of unsolvability of a regressive isol is defined as the Turing degree of any retraceable set belonging to it. If $A$ is a regressive isol then $\Delta_{A}$ will denote its degree of unsolvability. The notion of degree of unsolvability for a regressive isol is introduced in [6]. Among the results proved in [6] is the following: if two infinite regressive isols have a sum that is regressive, then their respective degrees are equal.

2. Intersections of regressive sets. Let $A$ and $B$ denote arbitrary regressive isols. We set

$$
A \cap B=([\alpha \cap \beta] \mid \alpha \in A \text { and } \beta \in B) .
$$

If either $A$ or $B$ is finite, then it is easy to see that $A \cap B$ is also finite. In this case, if $r=\operatorname{minimum}(A, B)$, then $A \cap B=(0, \cdots, r)$. If $Y$ is an isol with $Y \leqq A$ and $Y \leqq B$, then $Y$ is regressive. For such a $Y$ one can readily show $Y \in A \cap B$. Thus, if both $A$ and $B$ are infinite, then every finite $r$ will belong to $A \cap B$, and in this case one therefore has:

$$
\omega \subseteq A \cap B
$$

It will follow from the second and third theorems given below that there exists a variety of pairs $A, B$ of infinite regressive isols for which $A \cap B=\omega$. Moreover, it will be seen that one may choose such pairs of regressive isols to be cosimple and to have a regressive sum.

THEOREM 1. Let $A$ and $B$ be regressive isols with $A+B$ regressive. Let $Y \in A \cap B$. Then
1. $Y=U+V$ with $U \leqq A$ and $V \leqq B$,
2. $Y \leqq A+B$, and
3. $Y$ is regressive.

Proof. It is clear that statement 1 implies statement 2. Also, since any predecessor of a regressive isol is also regressive, statement 2 implies statement 3 . We therefore need only verify statement 1. This statement is clear if $Y$ is a finite isol. Let us assume, therefore, that $Y$ is an infinite isol. Let $Y=[\alpha \cap \beta]$ with $\alpha \in A$ and $\beta \in B$. Then both $\alpha$ and $\beta$ are infinite regressive sets. Select 
separated sets $\alpha^{\prime} \in A$ and $\beta^{\prime} \in B$. Then $\alpha^{\prime} \cup \beta^{\prime}$ is a set in $A+B$ and is therefore regressive; we let $c_{n}$ be a regressive function that ranges over $\alpha^{\prime} \cup \beta^{\prime}$. As both $\alpha$ and $\alpha^{\prime}$ belong to $A$, they are recursively equivalent, and, similarly, $\beta$ and $\beta^{\prime}$ are recursively equivalent. Let $\varphi_{0}$ and $\varphi_{1}$ be one-to-one partial recursive functions with $\alpha \subseteq \delta \varphi_{0}$, $\varphi_{0}(\alpha)=\alpha^{\prime}, \beta \subseteq \delta \varphi_{1}$, and $\varphi_{1}(\beta)=\beta^{\prime}$.

Define:

$$
\begin{aligned}
& \delta=\left(a \in \alpha \cap \beta \mid \varphi_{0}(\alpha)=c_{s}, \varphi_{1}(a)=c_{t} \text { and } s>t\right), \\
& \lambda=\left(a \in \alpha \cap \beta \mid \varphi_{0}(\alpha)=c_{s}, \varphi_{1}(a)=c_{t} \text { and } s<t\right) .
\end{aligned}
$$

It is easy to see that the sets $\delta$ and $\lambda$ are separated, and that their union is $\alpha \cap \beta$. In addition, we have,

(a) $\delta$ is a separated subset of $\alpha$, and

(b) $\lambda$ is a separated subset of $\beta$.

We shall verify only statement (a), for a similar argument may be given to verify (b). (Note that statement (a) implies $[\delta] \leqq A$, and statement (b) implies $[\lambda] \leqq B$.) To verify (a), assume $u \in \alpha$. We first compute the value of $\varphi_{0}(u) \in \alpha^{\prime}$. Assume $\varphi_{0}(u)=c_{s}$. Since $c_{n}$ is a regressive function, we can compute each of the values in the sequence $c_{0}, \cdots, c_{s}$. Some of these numbers may belong to the set $\beta^{\prime}$. We let

$$
\mu=\left(c_{0}, \cdots, c_{s}\right) \cap \beta^{\prime} .
$$

Since each of the numbers $c_{\imath}$ belongs to the set $\alpha^{\prime} \cup \beta^{\prime}$, and since $\alpha^{\prime}$ and $\beta^{\prime}$ are separated sets, it follows that we can effectively find all of the members of $\mu$. It now remains only to observe that $u \in \delta$ iff $u \in \varphi_{1}^{-1}(\mu)$, and the desired separability of the sets $\delta$ and $\alpha-\delta$ follows. This completes the proof of statement (a).

Let $U=[\delta]$ and $V=[\lambda]$. Then $U \leqq A$ and $V \leqq B$, and, lastly,

$$
\begin{aligned}
Y & =[\alpha \cap \beta] \\
& =[\delta \cup \lambda] \\
& =[\delta]+[\lambda] \\
& =U+V .
\end{aligned}
$$

We mention one corollary to Theorem 1; it concerns regressive isols that are cosimple. We recall that a cosimple isol is one that contains a cosimple set; it is known that not all sets belonging to such an isol need be cosimple. We shall make use of the following basic fact about sets with r.e. (recursively enumerable) complements (The "Reduction Theorem"): if two co-r.e. sets are disjoint, then they are separated. 
CoROLlary 1. Let $A$ and $B$ be cosimple regressive isols and $A+B$ be regressive. Let $\alpha \in A$ and $\beta \in B$ with $\alpha$ and $\beta$ cosimple sets. Then

$$
A+B=[\alpha \cup \beta]+[\alpha \cap \beta] .
$$

Proof. Let the sets $\delta$ and $\lambda$ be defined, as in the proof of Theorem 1, from the given sets $\alpha$ and $\beta$. Then $\delta$ is a separated subset of $\alpha$, and $\lambda$ is a separated subset of $\beta$. Since $\alpha$ and $\beta$ are cosimple, it follows that $\alpha-\delta$ and $\beta-\lambda$ are cosimple also. From the proof of Theorem 1 it follows that $\alpha \cap \beta=\delta \cup \lambda$ and that $\delta$ and $\lambda$ are separated sets. But $\alpha \cup \beta=(\alpha-\delta) \cup(\beta-\lambda)$. Since $\alpha-\delta$ and $\beta-\lambda$ are cosimple and disjoint, they are separated. Combining these facts gives the desired conclusion, as follows:

$$
\begin{aligned}
A+B & =[\alpha]+[\beta] \\
& =([\alpha-\delta]+[\delta])+([\beta-\lambda]+[\lambda]) \\
& =([\alpha-\delta]+[\beta-\lambda])+([\delta]+[\lambda]) \\
& =[(\alpha-\delta) \cup(\beta-\lambda)]+[\delta \cup \lambda] \\
& =[\alpha \cup \beta]+[\alpha \cap \beta] .
\end{aligned}
$$

A function $t_{n}$ from $\omega$ into $\omega$ is called T-retraceable if it is retraceable and has the property: if $\varphi$ is any partial recursive function then $\varphi\left(t_{n}\right)<t_{n+1}$ holds for all except at most finitely many values of $n$. A set is called T-retraceable if it is the range of a $T$-retraceable function, and an isol is called T-retraceable if it contains a $T$-retraceable set. The notion of $T$-retraceability has been used frequently in the literature on regressive isols. From [4] or [10], we know that there exist cosimple $T$-retraceable sets (and therefore also cosimple $T$-retraceable isols). The following result is Lemma 4 in [8].

Lemma 1 (Ellentuck). Let $\tau$ be a T-retraceable set. If $\alpha, \beta \leqq \tau$ are infinite recursively equivalent sets then $\alpha \cap \beta \neq \varnothing$.

TheOREm 2. Let $Y$ be a T-retraceable isol. Let $Y=A+B$ with both $A$ and $B$ infinite. Then $A \cap B=\omega$.

Proof. Note $\omega \subseteq A \cap B$, since both $A$ and $B$ are infinite. To show that $A \cap B$ contains only finite isols, assume $D \in A \cap B$. Let $D=[\delta]$ with $\delta=\alpha \cap \beta, \alpha \in A$ and $\beta \in B$. Let $\tau$ be a $T$-retraceable set in $Y$. Since $Y=A+B$ it follows that there will exist separated sets $\tau_{0}$ and $\tau_{1}$ such that $\tau=\tau_{0} \cup \tau_{1}, \tau_{0} \in A$ and $\tau_{1} \in B$. Thus, $\alpha$ and $\tau_{0}$, and likewise $\beta$ and $\tau_{1}$, are recursively equivalent. Let $\varphi_{0}$ and $\varphi_{1}$ be one-to-one partial recursive functions such that $\varphi_{0}(\alpha)=\tau_{0}$ 
and $\varphi_{1}(\beta)=\tau_{1}$. Then $\varphi_{0}(\delta)$ and $\varphi_{1}(\delta)$ are disjoint subsets of $\tau$, and, in addition, it is readily seen that they are recursively equivalent. It follows from Lemma 1 that each of these sets is finite. Hence $\delta$ is a finite set and $D$ is a finite isol.

REMARK. We note that every infinite regressive isol can be written as the sum of two infinite summands. It follows, therefore, from Theorem 2 that there exist infinite regressive isols $A$ and $B$ that have a regressive sum and for which $A \cap B=\omega$. Moreover, we may choose such regressive isols $A$ and $B$ to be cosimple, since there exist cosimple $T$-retraceable isols.

Proposition 1. Let $\alpha$ and $\beta$ be any two sets of numbers. Let $\alpha \cap \phi(\beta)$ be a finite set, for every one-to-one partial recursive function $\varphi$. Then $\mu \cap \lambda$ is a finite set, for every pair $(\mu, \lambda)$ such that $\mu$ is recursively equivalent to $\alpha$ and $\lambda$ is recursively equivalent to $\beta$.

Proof. Assuming $\mu$ is recursively equivalent to $\alpha$ and $\lambda$ is recursively :equivalent to $\beta$, we have $\mu=\varphi_{0}(\alpha)$ and $\lambda=\varphi_{1}(\beta)$ for some one-to-one partial recursive functions $\varphi_{0}$ and $\varphi_{1}$. Assume $\mu \cap$ $\lambda=\delta$. Then $\varphi_{0}(\alpha) \cap \varphi_{1}(\beta)=\delta$, with $\delta$ contained in the range of $\varphi_{0}$. This implies (since $\varphi_{0}$ is one-to-one) that $\alpha \cap \varphi_{0}^{-1} \varphi_{1}(\beta)=\varphi_{0}^{-1}(\delta)$. Since $\varphi_{0}^{-1} \varphi_{1}$ is one-to-one and partial recursive, it follows from our hypothesis that $\varphi_{0}^{-1}(\delta)$ is finite. Hence $\delta$ is also finite.

Since each isol $A$ is the collection of all sets that are recursively equivalent to any given member of $A$, we obtain the following corollary from Proposition 1.

Corollary 2. Let $A$ and $B$ be regressive isols. Let $\alpha \in A$ and $\beta \in B$ such that $\alpha \cap \varphi(\beta)$ is finite, for every one-to-one partial recursive function $\varphi$. Then $A \cap B \subseteq \omega$.

Lemma 2. Let $\alpha$ be any set which contains a simple set in its complement. Let $\varphi_{0}, \cdots, \varphi_{s}$ be any sequence of $s+1$ one-to-one partial recursive functions. Let $\lambda$ be any infinite r.e. set. Then there exists an infinite r.e. set $\mu \leqq \lambda$ such that $\phi_{i}(\mu) \cap \alpha$ is finite for each $i=0, \cdots, s$.

Proof. Let $\delta$ be a simple set contained in the complement of $\alpha$.

Define a set $\lambda_{0}$ in the following way: consider the set $\varphi_{0}(\lambda) \cap \alpha$. If this set is finite, let $\lambda_{0}=\lambda$. If this set is infinite, then the set $\varphi_{0}(\lambda) \cap \delta$ is also infinite. In this latter event, let $\lambda_{0}=\lambda \cap \varphi_{0}^{-1}(\delta)$. Then $\lambda_{0}$ is an infinite r.e. subset of $\lambda$, and, $\varphi_{0}\left(\lambda_{0}\right) \cap \alpha$ is finite (in 
fact, empty).

We may iterate the previous procedure, to obtain sets $\lambda_{s} \subseteq \cdots$ $\subseteq \lambda_{0}$ such that $\lambda_{i}$, for each $i=0, \cdots, s$, is an infinite r.e. set and $\varphi_{i}\left(\lambda_{i}\right) \cap \alpha$ is finite. Then $\mu=\lambda_{s}$ has the desired properties.

We will introduce next some special terminology that is used in our subsequent proof of Theorem 3. Let $F(x)$ be a total function, $\eta$ a set, and $m \in \eta$. We will interpret the expression " $F(m)=1$ stands alone in $\eta$ " as meaning $F(m)=1$ and $m$ is the only number in $\eta$ whose $F$-value is 1 . The expression " $F(m)=1$ does not stand alone in $\eta$ " we then interpret as meaning, either $F(m) \neq 1$ or else there exists a value $n \in \eta$ with $n \neq m$ and $F(n)=F(m)=1$. Also, we set $\omega^{+}=\omega-(0)$.

THEOREM 3. Let $A$ be an infinite regressive isol having the following property: there is a set $\alpha \in A$ such that $\alpha$ has a simple set contained in its complement. Then there exists a regressive isol $B$ with the following properties: $B$ is infinite, $A+B$ is not regressive, and $A \cap B=\omega$.

Proof. Let $\delta$ be a retraceable set with $\delta \in A$, and let deg $\delta$ denote the Turing degree of $\delta$. Then, $\Delta_{A}=\operatorname{deg} \delta$.

We shall define a retraceable function $b_{n}$, whose range $\beta$ will have the following properties: (1) $\beta$ is an isolated set, (2) $\beta$ is not Turing reducible to $\delta$, and (3) $\alpha \cap \varphi(\beta)$ is finite, for all one-to-one partial recursive functions $\varphi$. Let us assume such a set $\beta$ can be constructed, and let $B=[\beta]$. Then $B$ satisfies the desired requirements of the theorem. Clearly, $B$ will be an infinite regressive isol. The sum $A+B$ is not regressive, since the degrees $\Delta_{A}=\operatorname{deg} \delta$ and $\Delta_{B}=\operatorname{deg} \beta$ are unequal. Lastly, from Corollary 2 it follows that $A \cap B=\omega$.

For use in the definition of $b_{n}$, let $\left\{\phi_{i}\right\}$ be a listing of all oneto-one partial recursive functions, and let $\left\{F_{i}\right\}$ be a listing of all functions of one variable which are partial recursive in the characteristic function of $\delta$.

Define $b_{0}$ in the following way: set $\lambda=j\left(0, \omega^{+}\right)$, apply Lemma 2 , and let $\mu \cong \lambda$ be an infinite set with $\alpha \cap \varphi_{0}(\mu)$ finite. Set $b_{\text {n }}$ equal to the smallest number $m \in \mu$ such that $\varphi_{0}(m) \notin \alpha$ and $F_{0}(m)=$ 1 does not stand alone in $\mu$.

Assume the values $b_{0}, \cdots, b_{s}$ have already been defined. Define the value $b_{s+1}$ in the following way: set $\lambda^{\prime}=j\left(s+1, j\left(b_{s}, \omega^{+}\right)\right)$. Then $\lambda^{\prime}$ is an infinite r.e. set. Apply Lemma 2, and let $\mu^{\prime} \subseteq \lambda^{\prime}$ be an infinite set such that $\alpha \cap \varphi_{i}\left(\mu^{\prime}\right)$ is finite for each $i=0, \cdots, s+1$. Set $b_{s+1}$ equal to the smallest number $n \in \mu^{\prime}$ such that, 
(a) $\varphi_{i}(n) \notin \alpha$ for each $i=0, \cdots, s+1$, and

(b) $F_{s+1}(n)=1$ does not stand alone in $\mu^{\prime}$.

This completes the definition of the function $b_{n}$. It is easy to see that $b_{n}$ is a retraceable function. Let $\beta=\left(b_{0}, b_{1}, b_{2}, \cdots\right)$. It follows from our definition of $\beta$ that if $\varphi=\varphi_{i}$ is any one-to-one partial recursive function, then $\alpha \cap \varphi(\beta) \subseteq\left(b_{0}, \cdots, b_{i}\right)$, and, therefore, that $\alpha \cap \varphi(\beta)$ is finite. Since an infinite retraceable set is either recursive or isolated, it also follows, from the previous fact, that $\beta$ is an isolated set. This verifies properties (1) and (3). For (2), assume $\operatorname{deg} \beta \leqq \operatorname{deg} \delta$. Then there would exist a total function $F$, partial recursive in the characteristic function of $\delta$, such that $m \in \beta \Leftrightarrow F(m)=1$. Assume $F=F_{i}$. Then, in particular, we know that $b_{\imath}$ was defined so that $F\left(b_{i}\right)=1$ does not stand alone in $j(i, \omega)$. Yet $b_{i}$ is the only member of $\beta$ that belongs to $j(i, \omega)$. The contradiction which follows establishes (3), and thus completes the proof of the theorem.

Since each infinite cosimple isol contains a cosimple set, the following corollary is obtained directly from the previous theorem.

CoROllary 3. Let $A$ be an infinite cosimple regressive isol. Then there exists a regressive isol $B$ with the properties: $B$ is infinite, $A+B$ is not regressive, and $A \cap B=\omega$.

3. Invariantly $T$-retraceable isols. A regressive isol is called invariantly T-retraceable if it is infinite and every set belonging to it is $T$-retraceable. Invariantly $T$-retraceable isols were introduced and studied in [10]. It follows from (for example) an observation of Dëgtev in [4] that all cosimple invariantly $T$-retraceable isols will have the same degree of unsolvability, namely $0^{2}$. In this section we prove the existence of a pair of invariantly $T$ retraceable isols $A$ and $B$, having the following properties: $A$ and $B$ are cosimple, $A+B$ is not regressive, and $A \cap B=\omega$.

We shall use some properties of semirecursive sets. This notion was introduced by Jockusch in [9], and it is defined in the following manner: a set $\alpha$ is called semirecursive if there is a recursive function $\psi(x, y)$, of two variables, such that for all numbers $x$ and $y$ we have $\psi(x, y) \in(x, y)$, and $(x \in \alpha$ or $y \in \alpha) \Rightarrow \psi(x, y) \in$ $\alpha$. A recursive function $\psi(x, y)$ related to the set $\alpha$ by these properties is called a selector function for $\alpha$. It follows from [9, Theorem 3.2] that if a set is both cosimple and regressive then it is semirecursive. We shall use this fact in our subsequent proof of Theorem 4, to establish the nonregressiveness of a particular set constructed in that proof. We also use the following notation: 
if $\alpha$ is any set then $\bar{\alpha}$ will denote its complement.

THEOREM 4. There exist cosimple regressive isols $A$ and $B$ with the following properties:

(1) $A$ and $B$ are invariantly T-retraceable,

(2) $A+B$ is nonregressive, and

(3) $A \cap B=\omega$.

Proof. We use the technique of "movable markers". We shall construct sets $\alpha$ and $\beta$, and partial recursive functions $p(x)$ and $q(x)$, with the following properties:

(a) both $\alpha$ and $\beta$ are infinite and cosimple, $\alpha$ is a subset of the even numbers, and $\beta$ is a subset of the odd numbers,

(b) both $\alpha$ and $\beta$ are retraceable sets, and $p$ and $q$ are partial recursive functions that retrace $\alpha$ and $\beta$ respectively,

(c) the regressive isols $[\alpha]$ and $[\beta]$ are both invariantly $T$ retraceable,

(d) the set $\alpha \cup \beta$ is not semirecursive; and

(e) if $\phi$ is any one-to-one partial recursive function, then $\varphi(\alpha) \cap \beta$ is finite.

Let us assume that construction of sets $\alpha$ and $\beta$ with these properties can be carried out, and let $A=[\alpha]$ and $B=[\beta]$. Then $A$ and $B$ so defined will satisfy the requirements of the theorem. From (a), (b), and (c), both $A$ and $B$ are cosimple and invariantly $T$-retraceable isols. Combining (d), the fact that $\alpha$ and $\beta$ are separated sets, and [9, Theorem 3.2], we conclude that $A+B$ is a nonregressive isol. Lastly, combining (e) and Corollary 2, we obtain $A \cap B=\omega$.

For the construction, we use two kinds of movable markers on numbers: $\Lambda_{i}$ for the construction of $\alpha$ and $\Sigma_{i}$ for $\beta$. In addition, to obtain the property given in statement (d), we use one kind of tag on numbers, denoted by $*$. We let $\left\{\phi_{i}\right\}$ be an effective enumeration of the collection of all partial recursive functions of one variable, and we let $\left\{\psi_{i}\right\}$ be ian effective enumeration of the collection of all partial recursive functions of two variables. For each number $s, \varphi_{i}^{s}$ will denote the portion of $\varphi_{i}$ obtained at the end of the first $s$ stages of computation; $\psi_{i}^{s}$ is similarly related to $\psi_{i}$.

Stage 0. Attach markers $\Lambda_{0}$ to $0, \Lambda_{1}$ to $2, \Sigma_{0}$ to 1 , and $\Sigma_{1}$ to 3. Define $p(2)=p(0)=0$ and $q(3)=q(1)=1$.

Let $s \geqq 0$ and assume the construction has been carried out up to and including stage $s$. Let $\tau(s)$ be the number characterized by the property that $\Lambda_{0}, \cdots, \Lambda_{\tau(s)}$ and $\Sigma_{0}, \cdots, \Sigma_{\tau(s)}$ are the only markers 
that are presently attached to numbers. For each number $j \leqq \tau(s)$, let $a_{j}^{s}$ and $b_{j}^{s}$ denote the numbers that $\Lambda_{j}$ and $\Sigma_{j}$ are attached to, respectively.

Stage $s+1$. We proceed in the construction in four separate Steps A, B, C and $D$. In Step A we arrange for the invariant $T$ retraceability of both $[\alpha]$ and $[\beta]$; in Step $\mathrm{B}$, we work to insure that $\varphi(\alpha) \cap \beta$ is finite for each one-to-one partial recursive function $\varphi$; and in Step $\mathrm{C}$, we seek to make each recursive function $\psi$ fail in being a selector function for the set $\alpha \cup \beta$. Step $D$ is an auxiliary step introduced to tie together certain parts of Step C. We will say that a number is free at a given point in the construction if it is larger than every number which bears at that point a marker or has borne a marker at any previous point in the construction.

Step A. Let $0<j \leqq \tau(s)$. Consider the values of $a_{i}^{s}$ and $b_{i}^{s}$, and the functions $\varphi_{i}^{s}$, for $i=0, \cdots, j$. Define $\Delta_{\alpha}(j, e, f)$ to mean: $e, f \leqq j, \quad(\forall k \leqq j)\left[\varphi_{e}^{s}\left(\alpha_{k}^{s}\right)\right.$ is defined], $\varphi_{e}^{s}$ is one-to-one, and $(\exists k<$ $j)\left[\varphi_{f}^{s} \varphi_{e}^{s}\left(a_{k}^{s}\right)\right.$ is defined and has value $\left.\geqq \varphi_{e}^{s}\left(a_{j}^{s}\right)\right]$.

Define $\Delta_{\beta}(j, e, f)$ exactly as $\Delta_{\alpha}(j, e, f)$ with the one exception that within $\Delta_{\beta}(j, e, f)$ each occurrence of the letter $a$ is replaced by the letter $b$.

If $\Delta_{\alpha}(j, e, f)$ holds true, it implies the possible failure of $\alpha$, as thus far witnessed by its "approximate initial segment" $a_{0}^{s}, \cdots, a_{j}^{s}$, to be invariantly $T$-retraceable. If $\Delta_{\beta}(j, e, f)$ holds true, then there is a similar meaning which follows, with respect to $\beta$ and the values of $b_{0}^{s}, \cdots, b_{j}^{s}$. We consider separately two cases.

Case A1. For each number $j$ with $0<j \leqq \tau(s)$, and all numbers $e, f \leqq j$, neither $\Delta_{\alpha}(j, e, f)$ nor $\Delta_{\beta}(j, e, f)$ is true. In this event, proceed to Step B.

Case A2. There exist numbers $j$ with $0<j \leqq \tau(s)$ and numbers $e, f \leqq j$ for which either $\Delta_{\alpha}(j, e, f)$ is true or $\Delta_{\beta}(j, e, f)$ is true. Let $j_{0}$ be the smallest such number. Detach $\Lambda_{i}$ and $\Sigma_{i}$ for each $i$ such that $j_{0} \leqq i \leqq \tau(s)$. Detach $*$ from any number $m$ with $j_{0} \leqq$ $m \leqq \tau(s)$ which happens to bear a $*$. Let $2 r$ be the smallest even free number. Attach $\Lambda_{j_{0}}$ to $2 r$, and attach $\Sigma_{j_{0}}$ to $2 r+1$. Define $p_{A}^{s}=\left(\left\langle 2 r, a_{j_{0}-1}^{s}\right\rangle\right)$ and $q_{A}^{s}=\left(\left\langle 2 r+1, b_{j_{0}-1}^{s}\right\rangle\right)$, and then proceed to Step B.

Step B. Let $\tau^{-1}(s)$ be the number characterized by the property that at the completion of Step A, $\Lambda_{i}$ and $\Sigma_{i}$ for $i=0, \cdots, \tau^{A}(s)$, are 
the only attached markers. For each number $j$ with $0 \leqq j \leqq \tau^{A}(s)$, let $a_{j}^{A}$ and $b_{j}^{A}$ denote the numbers to which the markers $\Lambda_{j}$ and $\Sigma_{j}$, are, respectively, now attached.

Let $w$ be a number with $0<w \leqq \tau^{A}(s)$. Let $\square(w, e, z)$ express the statement: $\left[e \leqq w\right.$ and $z \leqq \tau^{A}(s)$ and $\varphi_{e}^{s}$ is one-to-one and $\varphi_{e}^{s}\left(\alpha_{w}^{A}\right)$ is defined and $\left.\varphi_{e}^{s}\left(a_{w}^{A}\right)=b_{z}^{A}\right]$. Then, if $\square(w, e, z)$ holds, we want to try to secure some changes in the eventual values of $a_{w}$ or $b_{z}$, or both, to deal with the possibility that $\varphi_{e}$ is actually one-to-one. We consider seperately two cases.

Case B1. For no number $w$ with $0<w \leqq \tau^{A}(s)$ do there exist numbers $e$ and $z$ with $\square(w, e, z)$ holding true. In this event, proceed to Step C.

Case B2. There exist numbers $w, e$, and $z$ with $0<w \leqq \tau^{A}(s)$ and with $\square(w, e, z)$ holding true. Let $\left\langle w_{0}, e_{0}, z_{0}\right\rangle$ denote the least such triple of numbers, relative to the lexicographic ordering of all triples of numbers. We consider separately three subcases.

Subcase B2.1. $w_{0}=z_{0}$ and $w_{0}$ currently does not carry a $*$ on account of $a_{w_{0}}^{A}$. Detach all markers $\Lambda_{i}$ with $w_{0} \leqq i \leqq \tau^{A}(s)$, and detach all markers $\Sigma_{j}$ with $w_{0}<j \leqq \tau^{A}(s)$. Remove $a *$ from all numbers $k$ with $w_{0}<k \leqq \tau^{A}(s)$ that are carrying a $*$. Let $2 r$ be the smallest even free number. Attach $A_{w_{0}}$ to $2 r$, and let $p_{B}^{s}=$ $\left(\left\langle 2 r, a_{w_{0}-1}^{A}\right\rangle\right)$ and $q_{B}^{s}=\varnothing$. Now proceed to Step C.

Subcase B2.2. $w_{0}=z_{0}$ and $w_{0}$ currently carries a $*$ on account of $a_{w_{0}}^{A}$. Detach all markers $\Lambda_{i}$ with $w_{0}<i \leqq \tau^{A}(s)$ and detach all markers $\Sigma_{j}$ with $w_{0} \leqq j \leqq \tau^{A}(s)$. Remove any * that may be attached to a number $k$ with $w_{0}<k \leqq \tau^{A}(s)$. Let $2 r+1$ be the smallest odd free number. Attach $\Sigma_{w_{0}}$ to $2 r+1$, and let $p_{B}^{s}=\varnothing, q_{B}^{s}=$ $\left(\left\langle 2 r+1, b_{w_{0}-1}^{A}\right\rangle\right)$. Now proceed to Step C.

Subcase B2.3. $w_{0} \neq z_{0}$. Set $y_{0}=$ maximum $\left(w_{0}, z_{0}\right)$. Detach all markers $\Lambda_{i}$ and $\Sigma_{i}$ with $y_{0} \leqq i \leqq \tau^{A}(s)$. Remove the * from any number $k$ with $y_{0} \leqq k \leqq \tau^{A}(s)$ that may carry a $*$. Let $2 r$ be the smallest even free number. Attach $\Lambda_{y_{0}}$ to $2 r$ and $\Sigma_{y_{0}}$ to $2 r+1$. Set $p_{B}^{s}=\left(\left\langle 2 r, \alpha_{y_{0}-1}^{A}\right\rangle\right)$ and $q_{B}^{s}=\left(\left\langle 2 r+1, b_{y_{0}-1}^{A}\right\rangle\right)$. Then proceed to Step C.

Step C. There are two aims in this step. The first is to insure that in the eventual determination of the sets $\alpha$ and $\beta$, the set $\alpha \cup \beta$ is not semirecursive. This we do by arranging that each $\psi_{i}(x, y)$ fail to be a selector function for $\alpha \cup \beta$. The second aim is to tie together the construction of values in the previous and pres- 
ent steps so that they may be continued further at the next stage; and this we do here and in an auxiliary Step D.

Let $\tau^{B}(s)$ be the number characterized by the property that $\Lambda_{i}$ and $\Sigma_{i}$ for $0 \leqq i \leqq \tau^{B}(s)$, are the only attached markers, at the conclusion of Step B. For each number $i$ with $0 \leqq i \leqq \tau^{B}(s)$, let $a_{i}^{B}$ and $b_{i}^{B}$ be the respective numbers to which the markers $\Lambda_{i}$ and $\Sigma_{i}$ are now attached.

Let $j$ be a number with $0<j \leqq \tau^{B}(s)$, and let $\nabla_{j}$ denote the statement: $\left[\psi_{j}^{s}\left(a_{j}^{B}, b_{j}^{B}\right)\right.$ is defined and $\left.\psi_{j}^{s}\left(a_{j}^{s}, b_{j}^{s}\right) \in\left(a_{j}^{B}, b_{j}^{B}\right)\right]$. The number $j$ may or may not bear a $*$. The significance of $a *$ on $j$ is that it indicates the current impossibility of $\psi_{j}(x, y)$ being a selector function for $\alpha \cup \beta$. Thus, we wish to "hang stars". The criteria for application of a $*$ to a number are introduced in this step. We consider separately two cases.

Case C1. There is a number $j$ such that $0<j \leqq \tau^{B}(s), j$ does not bear a $*$, and $\nabla_{j}$ holds true. Let $j_{0}$ be the smallest such number $j$. Let $2 r$ denote the smallest even free number.

Subcase C1.1. $\psi_{j_{0}}^{s}\left(a_{j_{0}}^{B}, b_{j_{0}}^{B}\right)=a_{j_{0}}^{B}$. Detach all markers $\Lambda_{j}$ and $\Sigma_{k}$ with $j_{0} \leqq j \leqq \tau^{B}(s)$ and $j_{0}<k \leqq \tau^{B}(s)$. Remove $*$ from each number $i$ for which $j_{0} \leqq i \leqq \tau^{B}(s)$ and $i$ currently bears a $*$. Attach $\Lambda_{j_{0}}$ to $2 r$, and set $p_{C}^{s}=\left(\left\langle 2 r, a_{j_{0}-1}^{B}\right\rangle\right)$ and $q_{\sigma}^{s}=\varnothing$. Tag $j_{0}$ with $\mathrm{a} *$ on account of $b_{j_{0}}^{R}$. Proceed now to Step D.

Subcase C1.2. $\psi_{j_{0}}^{s}\left(a_{j_{0}}^{B}, b_{j_{0}}^{B}\right)=b_{j_{0}}^{B}$. Detach all markers $\Lambda_{j}$ and $\Sigma_{k}$ with $j_{0}<j \leqq \tau^{B}(s)$ and $j_{0} \leqq k \leqq \tau^{B}(s)$. Remove $*$ from each number $i$ such that $j_{0} \leqq i \leqq \tau^{B}(s)$ and $i$ currently bears a $*$. Attach $\Sigma_{j_{0}}$ to the number $2 r+1$, and set $p_{\sigma}^{s}=\varnothing$ and $q_{\sigma}^{s}=\left(\left\langle 2 r+1, b_{j_{0}-1}^{B}\right\rangle\right)$. Tag $j_{0}$ with $a *$ on account of $a_{j_{0}}^{B}$. Proceed now to Step D.

Case C2. Either no number $j$ exists with $0<j \leqq \tau^{B}(s)$ and with $\nabla_{j}$ holding true, or if such a number $j$ does exist, then it carries $a *$. In either of these events, we proceed as follows. Let $2 u$ be the smallest even free number. Attach $\Lambda_{\tau} B_{(s)+1}$ to $2 u$ and attach $\Sigma_{\tau B(s)+1}$ to $2 u+1$. Set $p(x)=y$ in case $\langle x, y\rangle \in p_{A}^{s} \cup p_{B}^{s} \cup\left(\left\langle 2 u, a_{\tau B_{(s)}}^{B}\right\rangle\right)$, and set $q(x)=y$ in case $\langle x, y\rangle \in q_{A}^{s} \cup q_{B}^{s} \cup\left(\left\langle 2 u+1, b_{\tau^{B}(s)}^{B}\right\rangle\right)$. Then proceed to stage $s+2$.

Step D. Let the number $j$, be defined as in Case C1. Let $2 u$ be the smallest even free number. Attach $\Lambda_{j_{0}+1}$ to $2 u$ and attach $\Sigma_{j_{0}+1}$ to $2 u+1$. Set $p(x)=y$ in case $\langle x, y\rangle \in p_{A}^{s} \cup p_{B}^{s} \cup p_{O}^{s}$ or if $x=2 u$ and $y=$ the number to which $\Lambda_{j_{0}}$ is currently attached. Set $q(x)=$ $y$ in case $\langle x, y\rangle \in q_{A}^{s} \cup q_{B}^{s} \cup q_{C}^{s}$ or if $x=2 u+1$ and $y=$ the number 
to which $\Sigma_{j_{0}}$ is currently attached. We now proceed to stage $s+2$.

This completes our construction. We verify first the following two facts:

I. Each of the functions $p(x)$ and $q(x)$, as defined by the construction, is partial recursive; and

II. Each of the markers $\Lambda_{m}$ and $\Sigma_{m}$, for $m=0,1,2, \cdots$, eventually attains a permanently-held position on some particular number.

$R e$. I. Since numbers that enter into the domains of $p$ and $q$ are free just prior to their entry, it follows that both $p$ and $q$ are well-defined functions. Also, since the construction, as a whole, is effective, it follows that each of the sets of ordered pairs $\{\langle x, p(x)\rangle\}$ and $\{\langle x, q(x)\rangle\}$ is recursively enumerable. Hence both of the functions $p$ and $q$ are partial recursive.

$R e$. II. It clearly follows from the construction that the marker $\Lambda_{0}$ is permanently attached to the number 0 , and $\Sigma_{0}$ is permanently attached to the number 1 .

Let us assume that $n \geqq 0$ and that each of the markers $\Lambda_{i}$ and $\Sigma_{i}$, for $i=0, \cdots, n$, attains a permanent attachment to a corresponding number. We wish to show that each of the markers $\Lambda_{n+1}$ and $\Sigma_{n+1}$ will also become permanently attached. Let $s_{0}$ be a stage such that each of the markers $A_{n}$ and $\Sigma_{n}$ is found in its permanent position at the conclusion of stage $s_{0}$. In view of the construction, it follows that both markers $\Lambda_{n+1}$ and $\Sigma_{n+1}$ will be attached at the end of every stage $t \geqq s_{0}+1$. If these markers are never detached during a stage $t>s_{0}+1$ then they will be permanently attached at the conclusion of stage $s_{0}+1$, and the desired result follows. Let us, therefore, assume there does occur some detachment of $\Lambda_{n+1}$ or of $\Sigma_{n+1}$ during a stage $t>s_{0}+1$. This can occur in only three different ways, during each individual stage: either from an application of Case A2 (with $j_{0}=n+1$ ), or an application of Case B2 (with $n+1 \in\left(w_{0}, z_{0}\right)$ ), or an application of Case C1 (with $j_{0}=n+$ 1). In addition, once a detachment of one of the markers $A_{n+1}$ or $\Sigma_{n+1}$ is made during a particular stage $t>s_{0}+1$ of the construction, then, within that stage, the particular marker is immediately reattached to a new number.

Let us consider first the number of times one of the markers $\Lambda_{n+1}$ or $\Sigma_{n+1}$ can be detached, in the course of the construction past stage $s_{0}$, via an application of Case A2. By the procedure defined in Case A2, in particular, because of the one-to-one feature placed 
on the function $\varphi_{e}^{s}$, it follows that there can occur at most a finite number of detachments of either $\Lambda_{n+1}$ or of $\Sigma_{n+1}$ by an application of this case. Let $t_{0}$ denote a number such that $t_{0} \geqq s_{0}+2$, and neither $\Lambda_{n+1}$ nor $\Sigma_{n+1}$ moves because of an application of Case A2 during any stage $u$ with $u \geqq t_{0}$.

Let us consider next the number of times that a detachment of either of the markers $\Lambda_{n+1}$ or $\Sigma_{n+1}$ can occur at a stage $u \geqq t_{0}$ through an application of Case $\mathrm{C} 1$. Note that the procedure in Step B serves to preserve the attachment of a $*$ to $n+1$, at stage $t_{0}$ and at each following stage, except in Subcase B2.3 when $y_{0}=$ $w_{0}=n+1$. In view of this fact, and the procedure defined in Case C1, it is easy to check that (subsequent to stage $t_{0}$ ) each of the markers $\Lambda_{n+1}$ and $\Sigma_{n+1}$ can be detached, by an application of Case $\mathrm{C} 1$, at most a finite number of times, provided they suffer at most finitely many detachments via Subcase B2.3.

With respect to applications of Case B2, let us first, then, consider the number of times either of $\Lambda_{n+1}$ or $\Sigma_{n+1}$ could be detached via an application of Subcase B2.3, in a stage $u \geqq t_{0}$. By the oneto-one condition placed on $\varphi_{e}^{s}$ throughout Case B2, we see that there can occur at most finitely many such applications of Subcase B2.3. Let $u_{0}$ be the smallest number $u$ with the properties: $u \geqq t_{0}+1$ and neither $\Lambda_{n+1}$ nor $\Sigma_{n+1}$ moves by an application of either Subcase B2.3 or Case C1 at any stage subsequent to stage $u$. Then movements of $\Lambda_{n+1}$ or $\Sigma_{n+1}$, in a stage $t \geqq u_{0}+1$, will occur only within Subcase B2.1 or Subcase B2.2. We consider seperately two possibilities, based on the $*$-bearing status of the number $n+1$ at the conclusion of stage $u_{0}$.

Case 1. The number $n+1$ bears a $*$ at the conclusion of stage $u_{0}$. Then $n+1$ will retain a $*$ throughout all stages $t \geqq u_{0}+1$. Hence, by the construction, either (a) all the movements being considered are movements of $\Lambda_{n+1}$ under Subcase B2.1, or else (b) all of the movements are of $\Sigma_{n+1}$ under Subcase B2.2. In either of these events, because of the one-to-one condition placed on $\varphi_{e}^{s}$ throughout Case B2, it follows that there can be at most $n+2$ movements of either of the markers $\Lambda_{n+1}$ or $\Sigma_{n+1}$.

Case 2. The number $n+1$ does not bear a $*$ at the conclusion of stage $u_{0}$. Since we are in a situation where Case C1 will henceforth no longer apply toward moving the markers $\Lambda_{n+1}$ and $\Sigma_{n+1}$, it follows that throughout the construction beyond stage $u_{0}$, the number $n+1$ will always be without a $*$. Based upon the construction, this fact implies that all of the movements which we are now considering are movements only of the marker $\Lambda_{n+1}$, and more- 
over, that each such movement can occur only by an application of Case B2.1. It follows that the constraints placed upon the application of Case B2.1 make possible at most $n+2$ such detachments of the marker $\Lambda_{n+1}$.

In view of these remarks it follows that each of the markers $\Lambda_{n+1}$ and $\Sigma_{n+1}$ will achieve a permanent position of attachment after a finite number of stages in the construction. Hence, by induction, each of the markers $\Lambda_{j}$ and $\Sigma_{j}$, for $j=0,1,2, \cdots$, attains a permanently-held position on some particular number.

Definition. For each number $n$, we let $a_{n}$ and $b_{n}$ be, respectively, the terminal positions of the marker $\Lambda_{n}$ and of $\Sigma_{n}$. In view of the construction, it follows that $a_{n}=\lim _{s \rightarrow \infty} a_{n}^{s}$ and $b_{n}=\lim _{s \rightarrow \infty} b_{n}^{s}$. We also define: $\alpha=\left(a_{0}, a_{1}, a_{2}, \cdots\right)$ and $\beta=\left(b_{0}, b_{1}, b_{2}, \cdots\right)$.

It is easy to see, from the construction, that $\alpha$ is a subset of the even numbers and $\beta$ is a subset of the odd numbers. Hence $\alpha$ and $\beta$ are separated sets. To complete the proof, in view of our earlier remarks, it suffices to verify the following properties:

III. $\bar{\alpha}$ and $\bar{\beta}$ are recursively enumerable sets,

IV. $\alpha \cup \beta$ is not semirecursive, and therefore $\alpha \cup \beta$ is not regressive,

V. Each of the sets $\alpha$ and $\beta$ is retraceable and immune, and each of the isols $[\alpha]$ and $[\beta]$ is invariantly $T$-retraceable, and

VI. $\varphi_{t}(\alpha) \cap \beta$ is a finite set, for every one-to-one partial recursive function $\varphi_{t}$.

Re. III. Markers move, in the construction, only from previously-held positions up to free numbers. Therefore the set $\bar{\alpha}$ can be characterized in the following way:

$\bar{\alpha}=\left(x \mid(\exists n)(\exists s)(\exists u)(\exists w)\left[u<x<w\right.\right.$ and $\Lambda_{n}$ is attached to $u$ at the end of stage $s$, and $\Lambda_{n+1}$ is attached to $w$ at the end of stage $\left.s\right]$ ).

A similar expression exists for $\bar{\beta}$ in terms of the markers $\Sigma_{i}$. Hence both $\bar{\alpha}$ and $\bar{\beta}$ will be recursively enumerable sets.

Re. IV. Let us assume otherwise, and let $\psi_{n}$ be a selector function for $\alpha \cup \beta$. Then, by definition, $\psi_{n}(x, y) \in(x, y)$ for all $x$ and $y$, and if $x \in \alpha \cup \beta$ or $y \in \alpha \cup \beta$ then $\psi_{n}(x, y) \in \alpha \cup \beta$. Consider the number $n$ and the pair of numbers $\left\langle a_{n}, b_{n}\right\rangle$. Assume we are at the beginning of a stage $s$ in the construction where $\Lambda_{n}$ is already permanently attached to $a_{n}$ and $\Sigma_{n}$ is already permanently attached to $b_{n}$. If the number $n$ carries a $*$, then this $*$ could only have 
been received by an application of Case $\mathrm{C} 1$ prior to stage $s$. From the description of Case C1, it then follows that either $(\exists y)\left[\psi_{n}\left(a_{n}, y\right) \in\right.$ $\bar{\beta}]$ the odd numbers] or $(\exists x)\left[\psi_{n}\left(x, b_{n}\right) \in \bar{\alpha}\right]$ the even numbers]. Hence, in this case, $\psi_{n}$ could not be a selector function for $\alpha \cup \beta$. If the number $n$ does not carry a $*$, then $n$ will not carry a $*$ at any stage beyond stage $s$. (Since awarding it one would cause either $A_{n}$ or $\Sigma_{n}$ to move.) It follows therefore, in view of Step C, that $\psi_{n}\left(a_{n}, b_{n}\right) \notin\left(a_{n}, b_{n}\right)$, and hence, in this case also, $\psi_{n}$ could not be a selector function for $\alpha \cup \beta$.

Combining these facts we may conclude that $\alpha \cup \beta$ is not semirecursive. Then, by our earlier remarks about semirecursive sets, it also follows that $\alpha \cup \beta$ is not a regressive set.

$R e . V$. It follows directly from statement $I$ and from the definitions of the functions $p$ and $q$ and of the sets $\alpha$ and $\beta$ that the functions $p$ and $q$ are partial recursive and, respectively, retrace the sets $\alpha$ and $\beta$. (Thus, note that $p\left(a_{n+1}\right)=a_{n}$ and $q\left(b_{n+1}\right)=b_{n}$ follow from the construction.) So, $\alpha$ and $\beta$ are both retraceable sets. We know, from [7], that a retraceable set is either recursive or immune. If either $\alpha$ or $\beta$ is recursive, it would follow at once that $\alpha \cup \beta$ is semirecursive. By statement IV, therefore, both $\alpha$ and $\beta$ are immune sets. We prove next that the isols $[\alpha]$ and $[\beta]$ are each invariantly $T$-retraceable; and we shall do this only for $[\alpha]$, since a similar proof can be given for $[\beta]$. Let $\varphi_{e}$ be any oneto-one partial recursive function such that $\alpha \cong \delta \varphi_{e}$. We wish to verify that $\varphi_{e}(\alpha)$ is a $T$-retraceable set. First note that $\varphi_{e}\left(a_{n}\right)$ is a regressive function, since it is recursively equivalent to the retraceable function $a_{n}$. Let us consider any partial recursive function $\varphi_{f}$. Let $j=$ maximum $(e, f)$. It follows from the construction that Step $A$ is applied as often as is needed in order to obtain the property: $(\forall k)\left[\left(k>j\right.\right.$ and $\left.\left.\varphi_{e}\left(a_{k}\right) \in \delta \varphi_{f}\right) \Rightarrow \varphi_{f}\left(\varphi_{e}\left(a_{k}\right)\right)<\varphi_{e}\left(a_{k+1}\right)\right]$. Thus if $\varphi_{e}(\alpha)$ is a retraceable set, then it is a $T$-retraceable set. To see that $\varphi_{e}(\alpha)$ is actually a retraceable set, first note that $\varphi_{e}\left(a_{n}\right)$ is an eventually increasing function; for we may choose, in the previous discussion, the function $\varphi_{f}$ to be the identity function. Since $\varphi_{e}\left(a_{n}\right)$ is a regressive function, this fact gives the retraceability of the function $\varphi_{e}\left(a_{n}\right)$, and, therefore, of the set $\varphi_{e}(\alpha)$.

$R e$. VI. We wish to verify that if $\varphi_{t}$ is any one-to-one partial recursive function then $\varphi_{t}(\alpha) \cap \beta$ is a finite set. But, Step B of the construction is so designed that the only elements of $\alpha$ that $\varphi_{t}$ can map into $\beta$ are among the values $a_{0}, \cdots, a_{t}$. Hence $\varphi_{t}(\alpha) \cap \beta$ has cardinality $\leqq t+1$, and therefore is a finite set. This verifies statement VI, and completes the proof of the theorem. 
4. Concluding remarks. (1) The results of $\S \S 2-3$ leave open the question whether there is an isol theoretic characterization of the regressive intersection property $A \cap B \cong \Lambda_{R}$ (where $\Lambda_{R}$ is the set of regressive isols). Some of the intersection and separation properties considered in $\S 2$ of this paper are further studied in [12], yet no such characterization is yet at hand.

(2) One way of proving Lemma 1 is to note that if $\varphi_{e}$ is a one-to-one partial recursive function, $\tau$ a $T$-retraceable set, and $\lambda=$ $\left(x \in \tau \mid \varphi_{e}(x)\right.$ is defined and $\left.\varphi_{e}(x) \in \tau\right)$, then $\varphi_{e}$ restricted to $\lambda$ is the identity function except on a finite set. This observation suggests the possibility of proving Theorem 4 without an ad hoc construction, by choosing suitable disjoint subsets $\alpha$ and $\beta$ of a suitable $T$-retraceable set, with $\alpha$ and $\beta$ being representable as differences of r.e. sets. This approach, however, cannot be applied to prove Theorem 4 in view of the footnote on p. 574 of [11].

(3) Let us assume that $A$ and $B$ are infinite regressive isols, and $A+B$ is also regressive. Let $Y \in A \cap B$. From Theorem 1 it follows that $Y \leqq A+B$. It is natural to feel that perhaps in this case one also has either $Y \leqq A$ or $Y \leqq B$. This feature need not hold, and we simply state, without proof, the following result which implies this fact.

THEOREM. There exist cosimple, invariantly T-retraceable isols $A$ and $B$ with the following two properties: (1) $A+B$ is regressive, and (2) there is an isol $Y \in A \cap B$ such that neither $Y \leqq A$ nor $Y \leqq B$.

\section{REFERENCES}

1. K. I. Appel, There exist two regressive sets whose intersection is not regressive, J. Symbolic Logic, 32 (1967), 322-324.

2. K. I. Appel and T. G. McLaughlin, On properties of regressive sets, Trans. Amer. Math. Soc., 115 (1965), 83-93.

3. J. Barback, On the intersection of regressive sets, Notices Amer. Math. Soc., 22 (Feb. 1975), Abstract \#721-E1.

4. A. N. Dëgtev, Hypersimple sets with retraceable complements, Algebra i Logika, 10 (1971), 235-246. (Russian).

5. J. C. E. Dekker, Infinite series of isols, Proc. Sympos. Pure Math., Vol. 5, Amer. Math. Soc., Providence, R. I., (1962), 77-96.

6. - The minimum of two regressive isols, Math. Zeitschr., 83 (1964), 345-366.

7. J. C. E. Dekker and J. Myhill, Retraceable sets, Canad. J. Math., 10 (1958), 357-373.

8. E. Ellentuck, Universal cosimple isols, Pacific J. Math., 42 (1972), 629-638.

9. C. G. Jockusch, Jr., Semirecursive sets and positive reducibility, Trans. Amer. Math. Soc., 131 (1968), 420-436.

10. T. G. McLaughlin, Trees and isols, Part I, Rocky Mountain J. Math., 5 (1975), 401-418.

11. - On retraceable sets with rapid growth, Proc. Amer. Math. Soc., 40 (1973), 573-576. 
12. T. G. McLaughlin, A partial comparison of two conditions on the intersections of regressive sets, Archiv f. Math. Logik, 18 (1977), 159-167.

Received June 23, 1977.

Suny College

BUFFaLO, NY 14222

AND

TeXas Tech University

LUBвоCK, TX 79409 



\section{PACIFIC JOURNAL OF MATHEMATICS}

EDITORS

RICHARD ARENS (Managing Editor)

University of California

Los Angeles, California 90024

C. W. Curtis

University of Oregon

Eugene, OR 97403

C. C. MOORE

University of California

Berkeley, CA 94720
J. DUGUNDJI

Department of Mathematics University of Southern Californı Los Angeles, California 90007

R. Finn and J. Milgram Stanford University Stanford, California 94305

\section{ASSOCIATE EDITORS}

E. F. BECKENBACH

B. H. NEUMANN

F. WOLF

K. YoSHIDA

\section{SUPPORTING INSTITUTIONS}

UNIVERSITY OF BRITISH COLUMBIA
CALIFORNIA INSTITUTE OF TECHNOLOGY
UNIVERSITY OF CALIFORNIA
MONTANA STATE UNIVERSITY
UNIVERSITY OF NEVADA, RENO
NEW MEXICO STATE UNIVERSITY
OREGON STATE UNIVERSITY
UNIVERSITY OF OREGON

UNIVERSITY OF SOUTHERNEALIFORNIA STANFORD UNIVERSITY UNIVERSITY OF HAWAII UNIVERSITY OF TOKYO UNIVERSITY OF UTAH WASHINGTON STATE UNIVERSITY UNIVERSITY OF WASHINGTON 


\section{Pacific Journal of Mathematics \\ Vol. 79 , No. 1 \\ May, 1978}

Teófilo Abuabara, A remark on infinitely nuclearly differentiable

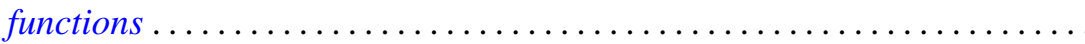

David Fenimore Anderson, Projective modules over subrings of $k[X, Y]$

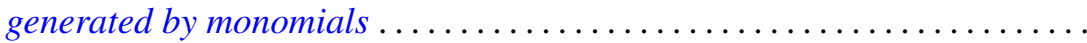

Joseph Barback and Thomas Graham McLaughlin, On the intersection of

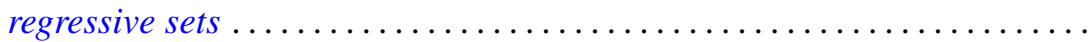

Murray Bell, John Norman Ginsburg and R. Grant Woods, Cardinal inequalities for topological spaces involving the weak Lindelof

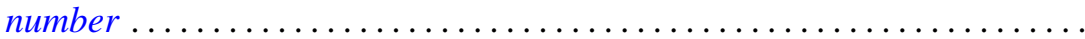

Laurence Richard Boxer, The space of ANRs of a closed surface ............

Zvonko Cerin, Homotopy properties of locally compact spaces at

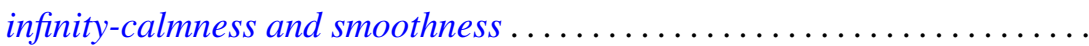

Isidor Fleischer and Ivo G. Rosenberg, The Galois connection between partial functions and relations..................................

John R. Giles, David Allan Gregory and Brailey Sims, Geometrical implications of upper semi-continuity of the duality mapping on a Banach

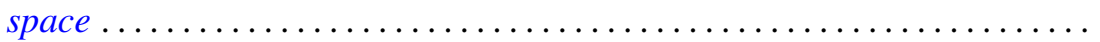

Troy Lee Hicks, Fixed-point theorems in locally convex spaces ............ Hugo Junghenn, Almost periodic functions on semidirect products of transformation semigroups ........................

Victor Kaftal, On the theory of compact operators in von Neumann algebras. II . . . .

Haynes Miller, A spectral sequence for the homology of an infinite

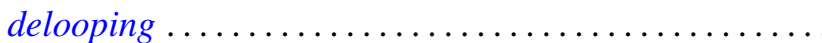

Sanford S. Miller, Petru T. Mocanu and Maxwell O. Reade, Starlike integral operators...

Stanley Stephen Page, Regular FPF rings ...............

Ghan Shyam Pandey, Multipliers for C, 1 summability of Fourier series ...

Shigeo Segawa, Bounded analytic functions on unbounded covering surfaces...

Steven Eugene Shreve, Probability measures and the C-sets of

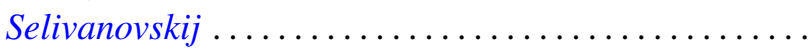

Tor Skjelbred, Combinatorial geometry and actions of compact Lie groups....

Alan Sloan, A note on exponentials of distributions.

Colin Eric Sutherland, Type analysis of the regular representation of a nonunimodular group.

Mark Phillip Thomas, Algebra homomorphisms and the functional

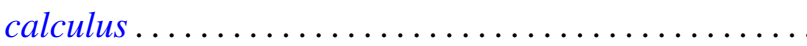

Sergio Eduardo Zarantonello, A representation of $H^{p}$-functions with

$0<p<\infty$. 